\title{
Erratum to cardiac arrest due to coronary embolism from a metastatic sarcoma recovered through aspiration thrombectomy
}

\author{
Min Gyu Kang, Kyehwan Kim, Hyun Woong Park, Jin-Sin Koh, Jeong Rang Park, Seok-Jae Hwang, \\ Jin-Yong Hwang \\ Division of Cardiology, Department of Internal Medicine, College of Medicine, Gyeongsang National University, Gyeongsang National University \\ Hospital, Jinju, Korea \\ Correspondence to: Jin-Yong Hwang. Department of Internal Medicine, College of Medicine, Gyeongsang National University, Gyeongsang National \\ University Hospital, 79 Gangnam-ro, Jinju 52727, Gyeongsangnam-do, Korea. Email: jyhwang@gnu.ac.kr.
}

doi: $10.21037 /$ jtd.2018.10.77

View this article at: http://dx.doi.org/10.21037/jtd.2018.10.77

Erratum to: J Thorac Dis 2018;10:E469-72

Cardiac arrest due to coronary embolism from a metastatic sarcoma recovered through aspiration thrombectomy

In the article that appeared on Page E469-E472, Vol 10, No 6 (June 2018) Issue of the Fournal of Thoracic Disease (FTD) (1), there is an error in the affiliation information. The correct affiliation should be "Division of Cardiology, Department of Internal Medicine, College of Medicine, Gyeongsang National University, Gyeongsang National University Hospital, Jinju, Korea".

The authors regret the error.

\section{References}

1. Kang MG, Kim K, Park HW, et al. Cardiac arrest due to coronary embolism from a metastatic sarcoma recovered through aspiration thrombectomy. J Thorac Dis 2018;10:E469-72.

Cite this article as: Kang MG, Kim K, Park HW, Koh JS, Park JR, Hwang SJ, Hwang JY. Erratum to cardiac arrest due to coronary embolism from a metastatic sarcoma recovered through aspiration thrombectomy. J Thorac Dis 2018;10(11):E791. doi: 10.21037/jtd.2018.10.77 